\title{
C. VON DER ERMORDUNG MATTEOTTIS ZU DEN LATERANVERTRÄGEN
}

(1924-1929)

16.

Aus der Rede des Genossen Bucharin über die Krise des faschistischen Regimes in Italien.

Aug. 1924

V. Weltkongreß der KOMINTERN

.....Es wäre vielleicht übertrieben, zu sagen (genaue Pressemeldungen über die Geschehnisse anläßlich der Ermordung Matteottis [10.6. 1924] liegen noch nicht $\left.\operatorname{vor}^{1}\right)$, daß dort [in Italien] schon eine neue Revolutionswelle beginnt, allgemeine Streiks usw., aber sicher ist, daß dort eine ganz neue Lage entsteht. Das zeigt allein, daß das kommunistische Organ „L'Unita“ in zwei Nummern auf der ersten Seite in Fettschrift die Parole „Nieder mit der Regierung der Mörder!“ brachte und daß Mussolini diese Zeitung nicht verboten hat. Das ist ein sicheres Zeichen, daß eine neue „Ära" begonnen hat. Wenn Mussolini erklärt, in diesem Moment gehe er nicht aus der Regierung - aber wenn diese Frage schon gestellt wird, so ist das ein „Zeichen der Zeit". Das ist die erste wichtige, tiefe innere Krise des Faschistenregimes in Italien.

Wenn morgen tatsächlich die bürgerliche Demokratie in Italien wieder siegt, so wird die neue "Demokratie" nicht dieselbe sein, wie sie 1920 war. Sie wissen, daß die sogenannte Sozialistische Partei in Italien eine große Massenpartei war. Die Arbeiterklasse fühlte sich frei, die bürgerliche Demokratie hatte ihre Blütezeit. Aber das Wesen der "Demokratie" begriffen die italienischen Arbeiter damals nicht. Die Sozialistische Partei war voller Illusionen. Die Arbeiter waren politisch naiv.....

Zwar haben auch wir, die Kommunisten, und nicht nur die Sozialdemokraten, manchmal die Lage zu einfach aufgefaßt und geglaubt: erst war Demokratie da, dann wird der Faschismus kommen und nach dem Faschismus kommt unbedingt die Diktatur des Proletariats. Das kann sein, das kann aber auch nicht sein. Wenn wir zum Beispiel Italien betrachten, so kann es sein, daß das Regime Mussolinis nicht unmittelbar von der Diktatur des Proletariats, sondern von einer neuen „Demokratie“ abgelöst werden wird, die gar nicht dieselbe sein wird, wie sie 1920 war, und daß schon unter der neuen „Demokratie“ sich die Diktatur des Proletariats vorbereiten wird.

Die Lage ist eben nicht so einfach, man kann nicht sagen, daß die Formel etwa so lautet: Demokratie, Buchstabe D; dann Faschismus, Buchstabe F; dann kommt unbedingt Revolution. Buchstabe R. Die Sache ist nicht so einfach. ... .

[Internationale Pressekorrespondenz Nr. 91 (August 1924), S. 1139]

1 Die Leiche des von Faschisten ermordeten sozialistischen Abgeordneten und Oppositionssprechers Giacomo Matteotti wurde erst am 12. 8. 1924 aufgefunden, die Tatsache dieser faschistischen „Rache" war aber schon in den Wochen vorher nicht zweifelhaft und hatte in der Kammer und Presse starke Erregung und heftige Kritik hervorgerufen, die Mussolini vorübergehend in die Defensive zwang. 
17.

\author{
Die Krise in Italien \\ Von Ruggiero Grieco (Rom).
}

....Der Faschismus, der Ende 1923 seine politische Bilanz mit dem Passivsaldo seiner innerlichen Krisen und der ungelösten wirtschaftlich-politischen Lage abgeschlossen hatte, war durch den Wahlsieg vom 6. April [1924] schwer kompromittiert, der den zwischen den Schwarzhemden und der Stimmung der proletarischen und kleinbürgerlichen Massen bestehenden Abgrund aufgezeigt hatte ${ }^{1}$. Die innere Zersetzung des Faschismus wurde anläßlich der Ermordung des sozialdemokratischen Abgeordneten Matteotti offenbar: Das Verbrechen an sich ist ein Beweis der Zersetzung der Partei, die sich in Italien an dex Macht befindet. In der Tat sind die für das Verbrechen Verantwortlichen alle in den leitenden Kreisen der herrschenden Partei und in den hohen Regierungskreisen gesucht und gefunden worden; und es ist noch nicht gesagt, daß uns die weitere Entwicklung der Dinge nicht noch weitere Einzelheiten zur Kenntnis bringt, die, wenn auch nicht unerwartet, doch sicherlich noch weitaus aufsehenerregender sein werden, als sie die öffentliche Meinung der Welt bisher gekannt hat.

Nach dem Verbrechen an Matteotti erlitt der Faschismus einen Schock, von dem es schien, daß er sich davon nicht mehr erholen werde. Es war die Schwäche der gegnerischen und der proletarischen Parteien, die es ihm gestattete, wieder Mut zu fassen. Tatsächlich ließ eine Untersuchung der voraussichtlichen Entwicklung der politischen Ereignisse und des Faschismus nicht in so kurzer Frist ein derartiges Geschehnis voraussehen, das dem Proletariat die Gelegenheit zu einer, wenigstens teilweise siegreichen, Offensive bieten konnte. Und selbst wenn ein solches Geschehnis vorhergesehen worden wäre, so hätten unsere Massen, die seit drei Jahren in schmerzhafter Weise zur Unbeweglichkeit verurteilt sind, nicht ohne eine fortschreitende Vorbereitung die Initiative ergreifen können. Das italienische Proletariat befindet sich in der Lage eines Rekonvaleszenten. Es kommt sicherlich wieder zu seinen alten Kräften, aber diese Wiederherstellung wird methodisch und ohne plötzliche Sprünge erfolgen, die ihm sehr schaden könnten. Der Faschismus bemerkte, daß ex trotz seiner 150000 Schwarzhemden Mitte Juni sehr schwach war, aber er bemerkte ebenso, daß er stärker ist als die Oppositionsparteien. ....

[Internationale Pressekorrespondenz Nr. 111 vom 22. August 1924, S. 1427]

1 Durch das im Juli 1923 gegen z. T. heftige Opposition mit Hilfe der bürgerlichen Rechten (Salandra, Giolitti u.a.) eingeführte neue Wahlgesetz war die Verhältniswahl abgeschafft und durch ein System ersetzt worden, das der Partei mit relativer Mehrheit mindestens 2 Drittel aller Parlamentssitze einräumte. Für die Kammerwahlen vom 6. April 1924 lieB Mussolini die Liste einer Regierungspartei aufstellen, die sich zu zwei Dritteln aus eingeschriebenen Faschisten, zu einem Drittel aus sympathisierenden Nationalisten, dekorierten Kriegsteilnehmern, Rechtsliberalen u.a. zusammensetzte. Sie erhielt $65 \%$ der Stimmen und damit 575 Mandate gegenüber 160 Mandaten der Opposition (davon 40 Popolari, 25 unitarische Sozialisten [unter ihnen Matteotti], 22 maximalistische Sozialisten, 17 Kommunisten). 
18.

Faschismus und „Kartell der Linken“

5. 9.1924

Von Augusto Piccini

Die Ereignisse politischer Natur, die sich im Monat August abgespielt haben, und die ganze Entwicklung der politischen Situation in den letzten Wochen lassen klar erkennen, daß in dem offenen Konflikt zwischen dem Faschismus und der großen Mehrheit der Bevölkerung Italiens, der in den Julitagen durch die Ermordung Matteottis ausgelöst wurde, keine Entspannung eingetreten ist, sondern daß im Gegenteil die politische Lage sich immer mehr zuspitzt und der Verfallsprozeß des Faschismus rasch seiner abschließenden Phase entgegengeht. . . . .

Ein weiteres Ereignis, das einen großen Teil der Chronik der letzten Wochen ausgefüllt und die öffentliche Meinung bewegt hat, ist die Auffindung der LeichelMatteottis gewesen. Die Umstände, unter denen der Kadaver des Märtyrers gefunden wurde, haben es der Oppositionspresse erlaubt, neue Hypothesen über die Organisation und die Durchführung des Verbrechens aufzustellen und ihrer Kampagne, die dahin geht, den Kreis der Mitschuldigen immer mehr zu erweitern, neue Argumente hinzuzufügen. Die Leichenfeier hätte zweifellos zu gewaltigen Massenkundgebungen Anlaß gegeben, aber es gelang der Regierung, diese zu hintertreiben, und die Leiche wurde bei Nacht und Nebel von der Stelle, wo sie von den Mördern oder ihren Helfershelfern verscharrt wurde, direkt in den Heimatort Matteottis überführt. Aufgrund der bisher angeführten Tatsachen kann man zweifellos behaupten, daß sich die Kräfteverhältnisse von Tag zu Tag zu ungunsten des Faschismus verschieben. Der Kampfwille der Arbeiter- und Bauernmassen wird zusehends stärker, das Proletariat gewinnt das Vertrauen in seine Kräfte wieder, der Klassenkampf beginnt neuerdings aufzulodern. In den Schichten der Kleinbourgeoisie wächst der Unmut über die Lage, in die sie durch die zweijährige Herrschaft Mussolinis versetzt wurde, und der Übergang dieser Schichten, die bisher das Kräftereservoir der faschistischen Organisation bildeten, in das Lager der Opposition, stellt eines der wesentlichsten Elemente der Krise des Faschismus dar. Auch der Staatsapparat beginnt, wie wir bereits gesehen haben, den Händen Mussolinis zu entgleiten.

Die Wirtschaftskrise ist keineswegs überwunden. Der schlechte Ausfall der Ernte, der steigende Kreditmangel, die unverminderte Arbeitslosigkeit, die verminderte Aufnahmefähigkeit des Innenmarktes sind die Hauptursachen der Krise. Die Stabilität des Staatshaushaltes erhält sich nur dank dem unerhörten Steuerdruck, der besonders auf den arbeitenden Klassen und der Kleinbourgeoisie lastet.

Alle diese Faktoren objektiven und subjektiven Charakters drängen zu einer Lösung der allgemeinen Krise der kapitalistischen Gesellschaftsordnung, die in der gegenwärtigen Phase als die Krise des faschistischen Regimes in akuter Form hervortritt. Für diesen ersten Schritt scheint nunmehr die Zeit reif zu sein.

[Internationale Pressekorrespondenz Nr. 116 rom 5. September 1924, S. 1503] 
19.

16.9. 1924

V. Weltkongreß der Komintern: Resolution über den Faschismus

Der Faschismus ist eine der klassischen Formen der Konterrevolution in der Verfallsepoche der kapitalistischen Gesellschaftsordnung, in der Epoche der proletarischen Revolution, im besonderen dort, wo das Proletariat den Machtkampf aufgenommen, aber weil es ihm an revolutionärer Erfahrung mangelt und weil keine führende revolutionäre Klassenpartei da ist, nicht verstanden hat, die proletarische Revolution zu organisieren und die Erhebung der Massen bis zur Errichtung der proletarischen Diktatur zu steigern.

Der Faschismus ist das Kampfinstrument der Großbourgeoisie gegen das Proletariat, zu dessen Niederwurf die legalen staatlichen Machtmittel nicht mehr ausreichen; er ist das außerlegale Kampfmittel der Großbourgeoisie zur Aufrichtung und Festigung ihrer Diktatur. Aber seiner sozialen Struktur nach ist der Faschismus eine kleinbürgerliche Bewegung. Seine Wurzeln hat er in den infolge der kapitalistischen Krise dem Untergang geweihten Mittelschichten sowie in den durch die Folgen des Krieges deklassierten Elementen (gewesenen Offizieren usw.) und teilweise sogar in den in ihren revolutionären Erwartungen enttäuschten und verbitterten Elementen des Proletariats.

Bei fortschreitendem Zerfall der bürgerlichen Gesellschaft nehmen alle bürgerlichen Parteien, insbesondere die Sozialdemokratie, einen mehr oder weniger faschistischen Charakter an, bedienen sich seiner Kampfesweise gegen das Proletariat und lösen so selbst die Gesellschaftsordnung auf, zu deren Erhaltung sie sich gebildet haben. Der Faschismus und die Sozialdemokratie sind die beiden Seiten ein und desselben Werkzeuges der großkapitalistischen Diktatur. Die Sozialdemokratie kann daher im Kampfe gegen den Faschismus niemals eine zuverlässige Bundesgenossin des gegen den Faschismus kämpfenden Proletariats sein. Infolge seiner inneren Widersprüche (Interessengegensätze zwischen Großbourgeoisie einerseits und den kleinbürgerlichen und proletarischen Elementen andererseits) gerät der Faschismus nach seinem Siege in einen politischen Bankrott, der zu seiner inneren Zersetzung führt (Italien). In einen ähnlichen Krisenzustand gerät er auch dort, wo er - ohne den formalen Sieg errungen zu haben - gezwungen ist, offen das großbürgerliche Regime zu unterstützen und $\mathrm{zu}$ verteidigen (Deutschland).....

[Internationale Pressekorrespondenz Nr. 119, Sondernummer vom 16. Sept. 1924, S. 1581]

20.

Das Attentat auf Mussolini und die kommunistische Partei Italiens Von Ercoli (Mailand).

Was ist die Wahrheit über das Attentat auf Mussolini [am 4. 11. 1925]? Das ist die erste Frage, die man sich stellen muß, und ist auch das Problem, auf das in diesen ersten Augenblicken eine genaue Antwort zu geben am schwersten ist. Die einzigen Meldungen, die vorliegen, sind jene der offiziellen Agentur der Regierung und der faschistischen Presse. Diese Meldungen, die übrigens sehr schwer kontrolliert werden können, gehen darauf aus, folgendes als sicher annehmen zu lassen: 
1. Daß der Versuch eines Attentats auf Mussolini vorlag, der von dem ehemaligen Abgeordneten der Unitarischen Sozialistischen Partei, Zaniboni, vollführt wurde, und daß dieser Versuch von der Polizei in dem Augenblicke vereitelt wurde, in dem bercits alle zur Durchführung notwendigen Handlungen vollbracht waren. Zaniboni sei in einem Hotel in Rom verhaftet worden, in einem Zimmer, von dessen Fenster man den Balkon sehen konnte, von dem sich Mussolini zeigen sollte, und habe ein Präzisionsgewehr und auch sonst alles vorbereitet, um ruhig zielen zu können, und im Augenblicke, in dem Mussolini sich zeigte, ihn niederschießen zu können. (Für den Erfolg bürgte die äußerste Genauigkeit der Waffe.)

2. Daß der Attentatsversuch die Folge eines Komplotts ist, das von Elementen der Oppositionsparteien geschmiedet wurde, im besonderen von Elementen, die an der Spitze der Freimaurer nud der Emigranten in Frankreich stehen, und daß das Komplott der Beginn einer breitangelegten Aktion war, mit der man gewaltsam das faschistische Regime niederschlagen wollte.

Die Wahrheit über den ersten Punkt ist am schwersten festzustellen. In den ersten Stunden nach der Verhaftung Zanibonis gaben einige faschistische Zeitungen Darstellungen, die von jenen abwichen, die in der Folge von der Polizei und vom Innenministerium verbreitet wurden. Es ist jedoch nicht unwahrscheinlich, daß, von Einzelheiten abgesehen, Zaniboni tatsächlich versucht hat, Mussolini zu töten.

Was den zweiten Punkt betrifft, ist er derjenige, der größere politische Bedeutung hat. Die Behauptungen der faschistischen Regierung und der Polizei über das Bestehen eines Komplotts zur Vernichtung des Regimes liefern dem Faschismus den besten Vorwand, um die Reaktion zu verstärken und neue Repressivmaßnahmen gegen die Kräfte der Opposition zu ergreifen. Man muß sich vor Augen halten, daß in den letzten Zeiten die Stellung des Faschismus an der Macht aus verschiedenen Gründen schlechter geworden war.

1. Vor allem wird die Wirtschaftslage immer schwerer, und dies ruft unter den zahlenmäßig stärksten Klassen dex Bevölkerung Unzufriedenheit hervor. Der Vertrag mit Amerika über die Schulden wird, wenn er abgeschlossen sein wird, zu einer neuen Verschlimmerung beitragen.

2. Die Bluttaten von Florenz ${ }^{1}$ haben im ganzen Lande tiefgehende Rückwirkung gehabt. Dies hat den Faschismus gezwungen, eine „pazifistische Offensive“ einzuleiten, jedoch ohne große Ergebnisse. Die öffentliche Meinung der Mittelklassen (des Kleinbürgertums) begann sich von neuem, wie nach dem Verbrechen an Matteotti, gegen den Faschismus einzustellen.

3. Es steht die Wiedereinberufung des Senats bevor, der die „faschistischsten“ Gesetze zu billigen haben wird, das heißt: die von der Kammer im verflossenen Sommer gebilligten Gesetze, die das verfassungsmäßige Regime gründlich abändern und eine Diktatur einführen, die nicht mehr bloß den Tatsachen, sondern auch dem Rechte nach der herrschenden Partei zukäme. (Gesetz gegen das Freimaurertum, Gesetz über die Kontrolle der Vereine, Preßgesetz, Abänderungen des Strafgesetzes,

1 Nachdem die faschistische Regierung am 20. Mai 1925 in der Kammer ein Gesetz betr. Überwachung und Verbot von Geheimgesellschaften (vor allem gegen die Freimaurer gerichtet) durchgesetzt hatte, war es am 3./4. Oktoher 1925 in Florenz zu einer aufsehenerregenden gewaltsamen „Jagd auf die Freimaurer" gekommen, durch die zahlreiche Oppositionelle verhaftet und mißhandelt wurden; vgl. Don Sturzo: Italien und der Faschismus. - Köln 1926, S. 187. 
Unterdrückung der Verwaltungsfreiheit in den Dorfgemeinden usw.). Um vom Senat die Billigung dieser Gesetze mit großerMehrheit zu erzielen, muß der Faschismus einen Druck auf ihn ausüben.

Wenn man sich diese Tatsachen vor Augen hält, versteht man, daß der Faschismus alles Interesse daran hat, an das Bestehen eines breitangelegten Komplotts glauben zu machen, und den Versuch Zanibonis aufs stärkste auszunützen, um eine neue Welle der Reaktion zu rechtfertigen. In der Tat ist man sofort an die Auflösung der Unitarischen (Einheits-) Sozialistischen Partei (Reformisten) geschritten [Anfang November 1925], deren Mitglied Zaniboni war, sowie zur militärischen Besetzung aller Freimaurerlogen und des Hauptsitzes der Freimaurer, wobei man zum Vorwand nahm, daß der General Cappello, ein hervorragender Freimaurer, der gleichfalls verhaftet wurde, ein Mitverschworener Zanibonis sei. Nicht genug damit: In Mailand sind gleichfalls die Arbeitskammer (Gesellschaftskartell) und der Sitz des Allgemeinen Arbeiterverbandes (Gewerkschaftskommission Italiens) besetzt worden, und die faschistischen Zeitungen fordern und kündigen neue Zwangsmaßnahmen an, wobei sie behaupten, daß alle Gruppen der Opposition in die Verschwörung verwickelt waren. Der Plan des Faschismus ist also klar genug.

Kann man wirklich glauben, daß ein Komplott bestanden habe, um in diesem Augenblicke den Faschismus niederzuschlagen, nachdem man Mussolini ermordet hätte? Alles geht dahin, dies als ausgeschlossen zu halten. Der Versuch Zanibonis ist eher als ein individueller Akt zu verstehen, der von einem aufrichtig antifaschistischen Element vollbracht wurde, das, nachdem es voll Überzeugung die politische Aktion der bürgerlichen Oppositionsparteien verfolgt hatte, sich zur Verzweiflung getrieben fand, heute, da die Wirklichkeit gezeigt hat, daß diese politische Aktion nicht eine antifaschistische Aktion gewesen ist, sondern statt zur Schwächung vielmehr zur wirklichen Stärkung des Faschismus beigetragen hat ${ }^{1}$.

Im Lager der Oppositionsparteien gibt es ohne Zweifel viele Elemente, die sich in der gleichen Gemütsverfassung befinden wie Zaniboni. Es handelt sich um politisch desorientierte Elemente. Niemand von ihnen hat jemals die Aktion der Oppositionsparteien in der ersten Periode nach dem Verbrechen an Matteotti kritisiert, als die verbrecherische Unzulänglichkeit dieser Aktion am offenbarsten war. Gegenüber der Kampagne der Kommunistischen Partei für die revolutionäre Mobilisierung und Vorbereitung der Massen haben sie sich immer skeptisch oder ablehnend verhalten. Dem Programme der Kommunistischen Partei, das darin bestand, eine Lösung der MatteottiKrise zu suchen und den Faschismus niederzuschlagen, indem im geeigneten Augenblicke die organisierte Kraft der werktätigen Klasse, des Proletariats der Städte und

1 Mit der "politischen Aktion" der bürgerlichen Opposition ist vor allem gemeint: Der nach dem Matteotti-Mord von den oppositionellen Fraktionen gefaßte Beschluß, an den Kammersitzungen nicht mehr teilzunehmen, der dazu führte, da $\beta$ die Opposition ein Jahr lang außerhalb der Kammer tagte und in Protesten und Denkschriften gegen die faschistische Regierung Stellung nahm (sogen. "Arentin"). Sie wurde dabei wesentlich unterstützt von der oppositionellen Presse, welche nicht aufhörte, Mussolini mit der "moralischen Frage" der faschistischen Verbrechen zu konfrontieren. Es gelang der Opposition aber nicht, den König und nennenswerte Kräfte der Armee gegen Mussolini zu gewinnen. Dieser trat vielmehr 1925 mit Presseverboten und den "faschistischen Gesetzen" zur Gegenoffensive an, welche die Opposition weitgehend lahmlegte und die förmliche Umwandlung der bisher beibehaltenen demokratischen Verfassung zur autoritären Diktatur einleitete. 
der Bauern, eingesetzt werden sollte, setzten sie romantische und völlig wirklichkeitsfremde Kampfpläne entgegen.

Es gab eine Zeit, in der sie eine Art monarchistischer Erhebung des Heeres oder eines Teiles des Heeres gegen die Schwarzhemden für möglich erachteten. Sie glaubten, auf die Unterstützung einiger Generale rechnen zu können, die aber auf das erste Angebot Mussolinis hin die Zusammenarbeit mit ihm annahmen (Badoglio). Nicht wenige glaubten, auf den Antifaschismus des Hofes und des Königs rechnen zu können, die aber ohne Zweifel mit dem Faschismus sehr fest verbunden sind. Mit einem Worte: Diese Elemente brachten die ganze Unsicherheit, Naivität und politische Unfähigkeit des Kleinbürgertums zum Ausdruck. Heute, da alle ihre Pläne gescheitert sind, da sich alle ihre Vorsätze als undurchführbar und unwirklich erwiesen haben, heute, da sich die Kräfte der bürgerlichen Opposition auflösen und der Faschismus sich rühmen kann, einen vollen Sieg über sie davongetragen zu haben - heute erscheint der individuelle Terror diesen Elementen als der einzige Ausweg. In Wirklichkeit ist er aber nichts anderes als der Beweis für ein erbärmliches politisches Versagen. Das Kleinbürgertum hat bei der Aufgabe, den Kampf gegen den Faschismus zu führen, jämmerlich versagt. . . . .

Es kann nicht anders sein, als daß ein Regime, das aus Blut und Gewalt hervorgegangen ist und sich mit Gewalt und Blut erhält, mit den gleichen Mitteln bekämpft werden muß. Statt das Attentat moralisch zu verurteilen, erklärt die Kommunistische Partei, daß sie sich mit allen jenen solidarisch fühlt, die gegen den Faschismus bis zum Ende kämpfen wollen und in diesem Kampfe die eigene Freiheit und das eigene Leben aufs Spiel setzen.

Politisch jedoch spricht die Kommunistische Partei eine Verurteilung der Handlung Zanibonis aus und betont, daß diese ein neues und letztes Zeichen des Zusammenbruches der Aktion der bürgerlichen Oppositionsparteien in ihrem Kampfe gegen den Faschismus darstellt. Die einzige Art und Weise, die Matteotti-Krise zum Niederschlagen des Faschismus auszunützen, bestand darin, die Macht der Arbeiterklasse zu mobilisieren und in den Kampf eingreifen zu lassen.

Statt dessen haben seit dem Tage der Ermordung Matteottis die bürgerlichen Antifaschisten und ihre Verbündeten (Reformisten, Maximalisten, Republikaner) in jeder Weise das Eingreifen und die Mobilisierung der Kräfte des Proletariats verhindert1. Sie verbreiteten die unsinnigsten Illusionen über die Möglichkeit einer Beseitigung des Faschismus auf friedlichem Wege, sie gebrauchten und unterstützten bei jeder Gelegenheit die Taktik der Gesetzmäßigkeit. Sie leisteten sogar dem Faschismus verschiedene Male Hilfe, um die Arbeiter an der Bewegung zu hindern. Auf diese Weise haben sie es dem Faschismus gestattet, sich zu retten, und sich selbst zum Zusammenbruche und zum Zerfall verurteilt. Heute, da sich alle Wege für sie als

1 Nach der Auffindung der Leiche Matteottis (12. 8. 24) hatten die Kommunisten versucht, einen Generalstreik zu organisieren, stießen dabei jedoch auf den Widerspruch der maximalistischen und unitaristischen Sozialisten, die (wohl mit Recht) fürchteten, daß eine solche Aktion Mussolini nur den Vorwand für ein gewaltsames Eingreifen der bereits mobilisierten faschistischen Milizen geben würde; vgl. Mannhardt, a.a.O., S. 355. In einer besonderen Kundgebung vom 20. 10. 1924 kritisierten die kommunistischen Abgeordneten die Unfruchtbarkeit der passiven Opposition und forderten den Übergang zur aktiven Opposition; vgl. Schulthess' Europ. Geschichtskalender, 1924, Bd. 65, S. 269. 
versperrt erweisen, sind die Handlungen individuellen Terrors seitens jener, die aufrichtig antifaschistisch sind, erklärlich, trotzdem aber nicht weniger unfruchtbar und vergeblich. ....

[Internationale Pressekorrespondenz Nr. 154 vom 13. November 1925]

21.

Vom „integralen Korporativismus“

Von Ruggero Grieco (Rom).

"Wir wollen die integrale (allumfassende) Korporation", schreien die faschistischen Gewerkschafter. Wißt ihr, was diese ungeheuerliche Maschine der integralen Gewerkschaften ist? Stellt euch vor, daß die faschistische Korporation der Industriellen und die faschistischen Korporationen der Techniker, der Verwalter und der Arbeiter von einem einzigen Sekretariat geleitet und kontrolliert werden sollen, das als höchstes Organ die Streitfälle erledigt und sie entscheidet, bevor sie nach außen hin zum Ausdrucke gelangen, und dann habt ihr den integralen Korporativismus. ....

Um zusammenzufassen: der faschistische Syndikalismus besteht nicht, und es besteht auch nicht der integrale Syndikalismus. Die Klassenzusammenarbeit des Faschisten enthüllt sich so, wie wir sie auch ohne schmückende Beiworte gekannt haben: als die Knechtung der werktätigen Klassen durch die Unternehmer. Aber die Industriellen haben dem Herm Rossoni ${ }^{1}$, ihrem Parteigenossen, einen schlimmen Streich gespielt. Aus Furcht vor der Entwicklung einer Kampagne für den integralen Syndikalismus haben sie die Bildung des Ministeriums der Korporationen dringend gefordert. Die Leitung aller Korporationen wird also dem Minister anvertraut, der nicht der Sekretär der Korporationen ist, sondern mehr an die großen Interessen des Bank- und Industriekapitals gebunden ist. Das Ministerium der Korporationen tötet den integralen Syndikalismus im Keime und sichert in eleganter Form die Kontrolle der faschistischen Arbeiterorganisationen durch die Industriellen. ....

[Internationale Pressekorrespondenz Nr. 80 vom 1. Juni 1926), S. 1282f.]

22.10 .1926

22.

Krisenerscheinungen des Faschismus in Italien Von Ercoli.

Die Einführung der Todesstrafe in Italien ist ein Akt der Furcht, der von dem ständigen Aufeinanderfolgen von Anschlägen auf das Leben Mussolinis bestimmt ist. In neun Monaten ist dreimal der Versuch gemacht worden, den "Duce“ zu töten. Das erste Attentat ist noch nicht aufgeklärt, und es bestehen noch Zweifel, ob es sich hier nicht um eine Provokation handelt. Beim zweiten und dritten Male aber ist

1 Edmondo Rossoni, Leiter des faschistischen Gewerkschaftsverbandes „Confederazione delle Corporazione Sindacali". 
Mussolini mit knapper Not davongekommen ${ }^{1}$. Da jetzt die Attentate einander in dieser Weise zu folgen beginnen, ist es sehr schwer zu behaupten, daß kein weiteres Attentat kommen wird. Man kann im Gegenteil mit Sicherheit annehmen, daß den ersten drei Attentaten noch andere folgen werden. Wer da wei $\beta$, was der Faschismus in Italien war und was er verübt hat, wird wohl verstehen, worauf sich diese Gewißheit aufbaut. Der Faschismus hat soviel Abscheu und soviel $\mathrm{Ha}$ gesät, daß es ganz natürlich ist, wenn aus den Massen eines erbitterten Volkes heraus „Rächer" erstehen. Das Volk Italiens ist überdies zu individuellen Handlungen geneigt. Die Einführung der Todesstrafe hat also eine Art „legislativen Terrors“ zum Zweck, um diese Drohung $\mathrm{zu}$ beseitigen. Es ist aber sehr zu bezweifeln, ob diese Maßnahme wirksam sein wird.

Nach dem dritten Attentat hat Mussolini in einer Ansprache an die Schwarzhemden zum ersten Male davon gesprochen, daß dem faschistischen Regime Gefahren drohen. Niemals hatte er vorher einen derartigen Satz gesagt. Immer, und besonders nach den Attentaten, hatte er Gewicht darauf gelegt, laut zu verkünden, daß das Regime "sicher", "gefestigt", „aufs höchste gefestigt", „unzerstörbar" usw. sei. In Wahrheit aber sind heute Kennzeichen einer fortschreitenden Verschärfung nicht nur der Lage Italiens im allgemeinen, sondern auch des Faschismus im besonderen unverkennbar.

Seitdem der Faschismus an die Macht gelangt ist, besonders aber in den letzten beiden Jahren, hat der Faschismus seine Politik ausschließlich im Interesse eines Blockes geführt, den das Finanzkapital, die Großindustriellen und die Agrarier um den Faschismus herum bildeten. Die Politik des Faschismus war nicht nur eine Klassenpolitik, sondern eine Politik im Dienste der am gierigsten schmarotzerhaften Schichten der Bourgeoisie. In der ersten Zeit schien diese Politik eine Entwicklung der Produktionstätigkeit zur Folge zu haben. Das jeglicher Fesseln ledige Kapital stürzte sich in industrielle Unternehmungen, wobei es hoffte, dort weit über das übliche Ausmaß hinausgehende Gewinne zu erzielen. Die Kapitalsanlagen wuchsen in einem außerordentlichen Ausmaße an.

Es handelte sich jedoch nur um eine Scheinblüte und um ein rein äußerliches Wachstum. Beide waren tatsächlich teilweise nur eine Folge der fortschreitenden Entwertung der Währung und andererseits erforderten sie, um wirksam zu werden, große Absatzmärkte. Aber der Binnenmarkt Italiens ist durch die Handlungen des Faschismus selbst - der, um die Staatsfinanz zu stabilisieren, und um den Reichtum zugunsten der plutokratischen Gruppen umzuschichten, den Mittelstand ins Elend gestürzt und die armen Klassen in einen verzweifelten Zustand gebracht hat, indem er den Industriellen freie Hand gab, den Arbeitslohn herabzusetzen und die Arbeitszeit zu erhöhen - auf das äußerste verarmt. Und Außenmärkte müssen für Italien erst gewonnen werden. Daher der Ursprung einer Krise, deren Kennzeichen immer klarer zutage treten, da der Faschismus gezwungen ist, um einen weiteren katastrophalen Sturz der Währung zu verhüten, Maßnahmen zu treffen, die die Vergeblichkeit des von ihm vollbrachten Stabilisierungswerkes verdecken sollen.

Nach vier Jahren faschistischer Herrschaft befindet sich heute Italien in einer unleugbar verschärften Wirtschaftslage. Die Hauptzweige der Industrie stehen vor

1 Am 7. April 1926 hatte in Rom eine Frau mit dem Revolver auf Mussolini geschossen, wobei dieser leicht verletzt worden war, und am 12. September 1926 war es in Rom zu einem erfolglosen Bombenattentat gegen Mussolini gekommen. Das Attentat bildete den Anlaß für die vom faschistischen Ministerrat am 2. Oktober 1926 beschlossene Wiedereinführung der Todesstrafe. 
einer Krise. Man sieht voraus, daß innerhalb weniger Monate ungeheure Massen von Arbeitern aufs Pflaster geworfen werden. Die Bankrotte setzen in einer katastrophalen Form ein. Die Enteignung des Mittelstandes zugunsten der Plutokratie geht systematisch weiter. Die Volksmassen werden zu einer immer größeren Einschränkung ihres Verbrauches gezwungen und müssen Schwarzbrot, wie in Kriegszeiten, essen. Was bleibt von der Sanierung, die der Faschismus versucht hat, übrig?

Zwei Dinge bleiben übrig: einerseits das System der Erpressung und der Einschüchterung, das dazu eingeführt wurde, um jegliche Bewegungsmöglichkeit der Arbeiterklasse zu unterdrücken, und andererseits die Redensarten und die Pläne imperialistischer Expansion. Diese beiden Dinge sind für den Faschismus eine Notwendigkeit.

Eine unbedingte Notwendigkeit ist die Versklavung der Arbeiterklasse und der Bauern durch die Unterdrückung der Klassenorganisationen, durch das Verbot der Streiks, durch die Abschaffung der Selbständigkeit der Gemeindeverwaltungen, der Pressefreiheit, der Versammlungsfreiheit usw. Nur die Versklavung der Arbeiterklasse gestattet es den Großindustriellen und den Großagrariern, ihre Stabilisierungspolitik zu entfalten. Aber all dies geht nicht glatt vor sich. Der Druck, der auf die Massen ausgeübt wird, ruft bei ihnen eine Rückwirkung hervor, eine sehr fühlbare Linksschwenkung. Was wird geschehen, wenn der Druck, wie dies unvermeidlich ist, noch vermehrt werden muß? Sicherlich sind augenblicklich nicht große Bewegungen in unmittelbarer Aussicht, aber immerhin sind die Aussichten für den Faschismus eher alles andere als beruhigend.

Eine zweite Notwendigkeit besteht darin, eine große nationalistische Phraseologie anzuwenden und große Expansionspläne zu verkünden. Es handelt sich dabei vor allem um eine Notwendigkeit, die sich daraus ergibt, daß sich für die Lage Italiens nicht innerhalb Italiens selbst Auswege finden. Aber es handelt sich nicht nur darum. Die imperialistische Prahlerei ist eine Notwendigkeit, die dem Faschismus durch seinen kleinbürgerlichen Ursprung selbst und durch die Notlage, in der er sich befindet, aufgedrängt wird. Während er eine Aktion entfaltet, die voll und ganz zugunsten des Großbürgertums ist, muß er die gesellschaftlichen Schichten, aus denen er hervorgegangen ist, an sich gebunden halten, indem er sie mit Redensarten berauscht macht. Aber wie lange kann dies dauern? Wie lange wird die nationalistische Berauschung dazu dienen können, die Auflösung der scheinbaren Einheit der bürgerlichen Kräfte, die der Faschismus in seinem Schoße und um sich herum geschaffen hat, zu vermeiden? Wie lange wird sie verhindern können, daß sich das Kleinbürgertum gegen eine Politik auflehnt, das ihm zugleich den Wohlstand und die Illusion der Macht entzieht?

Hier berühren wir eine der heikelsten Fragen, die Krise der Faschistenpartei selbst, eine Krise, die trotz der Bemühungen Mussolinis unerbittlich weitergeht. Nachdem der Faschismus allen oppositionellen Strömungen die Möglichkeit genommen hat, sich zu äußern, und nachdem er sich zur einzigen politischen Organisation des Landes gemacht hat, erlebt ex das Wiedererstehen von Oppositionen in seinem eigenen Schoße ...

Die Krise ist soweit gedieben, daß innerhalb der Faschistenpartei selbst jegliches System der Demokratie abgeschafft werden mußte. Die Partei wird auch in den örtlichen Organen von Funktionären geleitet, die von der Regierung ernannt sind. Die Mitgliederversammlungen sind jeglicher Funktion beraubt worden. Aber die Krise tritt dennoch in scharfen Formen zutage. In Triest gerieten in den Tagen des 
letzten Attentats die beiden Strömungen des Faschismus in einen bewaffneten Kampf untereinander, der zwei ganze Tage währte und die Verkündigung des Belagerungszustandes notwendig machte. In Rom unternahmen die Faschistenbanden der Opposition einen Angriff auf das Gebäude der Polizeiverwaltung . . . . .

Die Vorhut der Arbeiterklasse, die Kommunistische Partei Italiens, ist genügend gestählt, um auch angesichts der neuen Drohungen ihren Weg unbeugsam weiter zu verfolgen.

[Internationale Pressekorrespondenz Nr. 127 vom 22. Oktober 1926, S. 2179f.]

23.

Der „Ausnahmezustand“ in Italien

Von G. Aquila

Die Ereignisse der letzten drei Tage in Italien ${ }^{1}$ weisen eindeutig darauf hin, daß die Regierung Mussolinis und das faschistische Regime sich in einem ernsten, krisenhaften Moment befinden ..... .

Die wichtigsten Maßnahmen „zur Sicherung des Regimes“ sind die folgenden:

1. Auflösung aller nichtfaschistischen Parteien, Vereinigungen und Verbände.

2. Verbot der gesamten nichtfaschistischen Presse.

3. Einführung der zwangsweisen Internierung „aller derjenigen, die Handlungen begangen haben, die darauf abzielten, die soziale, wirtschaftliche oder nationale Ordnung des Staates zu stürzen, die Sicherheit zu gefährden und die Tätigkeit der Staatsgewalt zu durchkreuzen und zu behindern, sowie derjenigen, die den Entschluß kundgetan haben, etwas Derartiges zu tun. "(!)

4. Einrichtung einer faschistischen politischen Polizei bei jedem faschistischen Milizkommando.

5. Ungültigkeitserklärung aller Auslandspässe; strenge Strafen für jeden, der das Land ohne $\mathrm{PaB}$ zu verlassen versucht, und für diejenigen, die ihm dabei behilflich sind: Verpflichtung zum Waffengebrauch, um ein unerlaubtes Überschreiten der Grenze zu verhindern.

Außer diesen „Maßnahmen“ ist im Ministerrat vom 5. November das sogenannte "Gesetz zum Schutze des Staates" beschlossen, d.h. das Gesetz, das die Todesstrafe einführt. Die wichtigsten Bestimmungen dieses Gesetzes sind:

1. Todesstrafe für diejenigen, die einen Anschlag auf das Leben, die Unversehrtheit oder die persönliche Freiheit des Königs oder des Regenten, der Königin, des Kronprinzen oder des Ministerpräsidenten verüben.

2. Todesstrafe für diejenigen, die eine Handlung begehen, die darauf abzielt, den Staat in die Abhängigkeit des Auslandes zu bringen(?!) oder die Unabhängigkeit des

1 Am 31. Oktober 1926 war es in Bologna zu einem neuen Revolverattentat auf Mussolini gekommen, dem dieser nur knapp entging. Der 15jährige Täter, Sohn eines Buchdruckers in Bologna wurde auf der Stelle gelyncht. Die faschistische Regierung nahm den Vorfall zum Anlaß, um am 5. November durch den Ministerrat eine Reihe schwerwiegender „Sicherheitsmaßnahmen" zu ergreifen, die eine wesentliche Etappe zur diktatorischen Umbildung des faschistischen Systems darstellten und im folgenden von der INPREKORR im wesentlichen zutreffend aufgezählt wurden. 
Staates zu bedrohen; für diejenigen, die politische oder militärische Geheimnisse betreffend die Sicherheit des Staates verraten oder sich in den Besitz solcher Geheimnisse setzen.

3. Todesstrafe für diejenigen, die Handlungen begehen, die darauf abzielen, die Bürger zu bewaffnetem Aufstand gegen die Staatsordnung aufzureizen; für diejenigen, die an einem Aufstand teilnehmen; und für diejenigen, die zum Bürgerkrieg aufreizen.

4. Die auf eine der vorerwähnten "Verbrechen " abzielende Verabredung wird mit 15 bis 30 Jahren Zuchthaus bestraft; ihre Verteidigung in der Presse mit Zuchthaus von 5 bis 15 Jahren.

5. Die Neubildung aufgelöster Parteien, Vereinigungen und Verbände wird, selbst wenn sie unter einem anderen Namen exfolgt (!), mit Zuchthaus bis zu 10 Jahren bestraft. Die Mitglieder solcher Organisationen werden mit Zuchthaus bis zu 5 Jahren bestraft.

6. Der italienische Staatsbürger, der innerhalb des Staatsgebietes falsche, übertriebene oder tendenziöse Nachrichten über die innere Lage des Landes verbreitet oder eine den nationalen Interessen schädliche Tätigkeit entfaltet, wird mit 5 bis 15 Jahren Zuchthaus bestraft.

7. Ein Italiener oder Ausländer, der im Auslande die vorerwähnten „Verbrechen “ begeht, wird ,in contumaciam" (in Abwesenheit) durch die italienischen Gerichte abgeurteilt.

8. Alle oben erwähnten „Verbrechen“ werden von Sondergerichten abgeurteilt, die unter dem Vorsitz eines Generals der Armee, der Marine, der Luftschiffahrt oder der Miliz stehen, und sich aus fünf Offizieren der faschistischen Miliz zusammensetzen. Im Prozeß wird das in Kriegszeiten geltende Militärstrafgesetzbuch angewandt......

[Internationale Pressekorrespondenz Nr. 136 rom 9. November 1926, S. 2358]

26.4. 1927

24.

Mussolinis „Magna Charta der Arbeit"

Von G. Aquila

Über ein Jahr hat man von der "Magna Charta der Arbeit“ in Italien geredet". Man hat gesagt, sie werde der "faschistischen Revolution" die Krone aufsetzen. Mussolini hatte in seiner gewohnten Bescheidenheit erklärt, sie werde eine neue Epoche in der Weltgeschichte eröffnen, indem sie an Stelle der von einem gewissen Karl Marx künstlich konstruierten und durch nichts begründeten Lehre vom Klassenkampf endgültig das Prinzip der „harmonischen Zusammenarbeit aller Faktoren der

1 Am 21. April, der von der Mussolini-Regierung anstelle des sozialistischen 1. Mai zum Feiertag der Arbeit erhoben worden war, hatte der Große Rat der faschistischen Partei die "Carta di Lavoro" verabschiedet, welche die Rechte und Pflichten der faschistischen Syndikate unter der Kontrolle des Staates regelte: unter der Devise, daß die Interessen der Nation denen des einzelnen vorausgehen, waren die Tariffreiheit ... überlieferte gewerkschaftliche Errungenschaften beseitigt und den Arbeitgebern als verantwortlichen Leitern der Produktionsstätten verstärkte Führungsrechte eingeräumt worden. 
Produktion" setzen und somit die Irrlehre vom Sozialismus und den Spuk des Kommunismus ein für allemal aus der Welt schaffen wird. Die faschistische Presse hat die Worte des „Duce“ aufgefangen und mit den schönsten Farben die Perspektiven der Zukunft gemalt: Italien, das faschistische Italien, der italienische „faschistische Staat“ wird wieder an der Spitze der Zivilisation der Menschheit marschieren, und die ganze Welt wird ungeteilt Mussolini, dem Schöpfer und Duce des Faschismus und Erlöser der Menschheit, huldigen. So wird - die „Magna Charta der Arbeit“ - das Evangelium der nächsten Jahrhunderte sein ...

Das ist also die welterschütternde und welterneuernde „Magna Charta der Arbeit“, die "Vollendung der faschistischen Revolution", der "Grundstein zum faschistischen Staat", die "Eröffnung einer neuen Epoche in der Geschichte der Menschheit" usw. usw.! Ein nur allzu ungeschickter und tölpelhafter Versuch zur Verschleierung der kapitalistischen Reaktion, deren brutalste Verkörperung der Faschismus ist.

Dennoch wird die "Magna Charta" Mussolinis einen großen Dienst erweisen: Wenn es in Italien und im Auslande noch einen Arbeiter gab, der sich durch die volltönenden Phrasen der faschistischen und bürgerlichen Presse betören ließ und sich irgendwelchen Illusionen hingab, so werden ihn die „Magna Charta der Arbeit" und die Praxis des "faschistischen Gewerkschaftsstaates" eines Besseren belehren. Die klassenbewußten, revolutionären italienischen Arbeiter, die bereits zum systematischen Wiederaufbau der von der faschistischen Regierung "aufgelösten “ und von ihren reformistischen Führern feige verlassenen Gewerkschaftsorganisationen geschritten sind, werden in ihrer unerschütterlichen Arbeit durch Mussolinis „Magna Charta " nur gestärkt.

[Internationale Pressekorrespondenz Nr. 45 vom 26. April 1927, S. 924f.]

25.

Der Weg des italienischen Kapitalismus

Von E. Varga

Die Entwicklung des italienischen Kapitalismus im letzten Jahrzehnt zeigt einige besonders interessante Momente. Italien ist die Großmacht Europas, die am spätesten in die moderne kapitalistische Entwicklung eingetreten ist, trotzdem aber in kürzester Zeit einen ausgesprochen imperialistischen Charakter angenommen hat. Die offene Forderung einer Neuverteilung der Erde und offene Kriegsrüstungen zu diesem Zweck charakterisieren am besten den italienischen Kapitalismus.

Die zweite Besonderheit in der Entwicklung des italienischen Kapitalismus ist die Tatsache, daß die Diktatur der Bourgeoisie hier die besondere Form des Faschismus angenommen hat! Es taucht die Frage auf, inwiefern dieses Regierungssystem die Entwicklung der italienischen Wirtschaft - gegenüber jener anderer Länder hemmt oder fördert? Wir wollen hier, das Ergebnis vorausnehmend, sagen: Die Tatsache des Bestehens des faschistischen Regimes hat keine irgendwie bedeutende Differenz in der Entwicklung der italienischen Wirtschaft in der Nachkriegszeit nach sich gezogen, wenn wir diese mit Frankreich, dessen Verhältnisse am nächsten liegen, vergleichen. Ebenso wie Frankreich ist es auch Italien gelungen, die Valuta zu stabilisieren und die Kriegsfolgen bis zu einem gewissen Grade zu überwinden. Die 
Folge des Bestehens des faschistischen Systems ist aber, daß diese Reorganisation der kapitalistischen Wirtschaft in Italien in viel stärkerem Maße auf Kosten der Arbeiterschaft vor sich ging als in Frankreich oder Deutschland. Während im übrigen Westeuropa die reale Lebenshaltung des Volkes im allgemeinen und wahrscheinlich auch der Arbeiterklasse im besonderen nicht wesentlich schlechter als vor dem Kriege ist, deuten sichere Zeichen, die wir später entwickeln werden, darauf hin, daß das faschistische Regime eine absolute Verschlechterung der Lage der Arbeiterklasse nach sich gezogen hat. ....

[Internationale Pressekorrespondenz Nr. 78 vom 4. August 1927]

24.2.1928

26.

Mussolinis Verfassungsreform

Arn 20. Februar tagte der italienische Ministerrat unter dem Vorsitz Mussolinis. Mussolini legte dem Ministerrat verschiedene Gesetzentwürfe vor, darunter den neuen Wahlgesetzentwurf, der nach seiner Äußerung „dem Ziel des weiteren Aufbaus des faschistischen Staates und einem organischen Plan, dem Kriterium der faschistischen Einheit entsprechen wird".....

Der Zweck, den Mussolini mit diesem Gesetzentwurf verfolgt, liegt klar auf der Hand: Alle oppositionellen Parteien in Italien sollen vernichtet werden, nur eine einzige politische Partei soll existieren, die in allem und jedem der faschistischen Regierung, d.h. den Großkapitalisten und den Großgrundbesitzern dient. Dies hat das offiziöse Organ der faschistischen Regierung im November 1927, als der Große Faschistenrat das neue Wahlgesetz beschlossen hatte, auch offen eingestanden. Es schrieb damals, daß „die Verfassungsreform das Ende des Parlaments im traditionellen Sinne des Wortes bedeuten wird".

Das neue Gesetz ist ein Ausdruck dessen, daß der Faschismus kein parlamentarisches Regime ertragen kann. Ein parlamentarisches Regime setzt die Existenz von oppositionellen Parteien und die Existenz einer oppositionellen Presse voraus. Der Faschismus, der in den letzten zwei Jahren alle wirtschaftlichen Kräfte des Landes, der Bürokratie, der bewaffneten Gewalt in den eigenen Händen konzentriert, sich immer mehr mit dem Kapitalismus identifiziert hat, die faschistische Partei, die voll und ganz unter die Leitung der Industriellen und Agrarier geriet und infolgedessen diejenigen kleinbürgerlichen Schichten, die ihre ursprüngliche soziale Basis bildeten, notwendigerweise weggestoßen hat, kann aber den werktätigen Massen keine solche „demokratischen Rechte" mehr gewähren. Der Faschismus muß jede direkte oder indirekte Einmischung der werktätigen Massen in das politische Leben des Lancies zu verhindern versuchen, da jede solche Einmischung auf eine Gefährdung der faschistischen "Stabilisation" hinauslaufen könnte. Das ist der politische Sinn der neuen „Verfassungsreform“.

[Internationale Pressekorrespondenz Nr. 19 vom 24. Februar 1928, S. 396f.] 
27.

Zum VI. Jahrestag des Faschismus in Italien

30.10 .1928 Von G. Aquila

..... Sechs Jahre faschistischen Regimes haben in Italien eine gewaltige Umwälzung vollbracht. Nicht die "Umwälzung", die die faschistische Presse auch heute noch als eine vollbrachte oder zumindest zum Teil bereits verwirklichte Tat in die Welt hinauszulügen bestrebt ist, allerdings ohne mit ihrer Lüge bei irgendeinem halbwegs normalen Menschen Glauben zu finden: die "Einigung der gesamten Nation " auf Grund des - Klassenfriedens. Nein : das Gegenteil davon. Die sechs Jahre faschistischen Regimes haben den Klassenkampf in Italien in einer noch nie dagewesenen Schärfe zugespitzt und ihn auf eine höhere Stufe gebracht: sie haben nicht nur die Spaltung des italienischen Volkes in zwei voneinander durch eine unüberbrückbare Klaft getrennte Lager beschleunigt, sondern sie haben auch jedem in Italien Lebenden diese Spaltung bewußt gemacht: hie die kleine Minderheit der durch den Faschismus regierenden Ausbeuter - hie die übergroße Mehrheit der mittels des Faschismus geketteten Ausgebeuteten. In den sechs Jahren seiner Herrschaft ist das faschistische Regime im Bewußtsein aller Italiener ein Synonym des kapitalistischen Regimes, der kapitalistischen Gesellschaftsordnung geworden.

Darin wurzelt seine Schwäche, die ihm den Stempel des nahenden Todes aufdrückt: darin besteht aber gleichzeitig auch seine gegenwärtige Kraft, die erst durch das revolutionäre Proletariat gebrochen werden muß.

Der Faschismus ist eine Gewaltherrschaft der Bourgeoisie. Das ist er indes nur im allgemeinen, insofern er die Profitgewinnung für alle Ausbeuter gegenüber allen Ausgebeuteten sichert oder zu sichern bestrebt ist. Wenn wir aber näher zuschauen, wenn wir die Politik der faschistischen Regierung in allen ihren Einzelheiten untersuchen, dann erkennen wir, daß der Faschismus im besonderen die Gewaltherrschaft des Großkapitals, noch genauer, die Herrschaft der Hochfinanz und des IudustrieGroßkapitals im Bündnis mit den Agrariern ist. Praktisch bedeutet das so viel, daß der Faschismus zwar gegen die Ausgebeuteten allen Ausbeutern in Stadt und Land das Profitmachen sichert, innerhalb des Lagers der Ausbeuter aber vor allem die Interessen des Großkapitals wahrt - auch gegen die Interessen der unteren und mittleren Schichten der Bourgeoisie selbst. Mit anderen Worten: Er fördert die Akkumulation des Großkapitals mit allen Mitteln auch auf Kosten des kleinen und mittleren Kapitals.

Die logische Folge dieser offensichtlichen Tatsache ist, daß diese unteren und mittleren Schichten der italienischen Bourgeoisie, ja, zuweilen sogar auch manche großkapitalistischen Gruppen, deren Interessen ebenfalls den Interessen der entscheidenden Schichten des Großkapitals geopfert werden, heute schon alles eher als „begeisterte" Faschisten sind.

Auf diese Erscheinung baut die italienische Sozialdemokratie aller Schattierungen (Maximalisten, Turatianer) ihre Politik. Sie geht zwar noch einen "kleinen" Schritt weiter und stellt die unsinnige Behauptung auf, daß der Faschimus die Herrschaft eines kleinen Häufleins verwegener Verbrecher sei, das alle Klassen: sowohl Proletariat und Kleinbürgertum in Stadt und Land als auch die gesamte Bourgeoisie "unterdrückt und ausbeutet“, aber - auf solche „Kleinigkeiten“ kommt es unseren sozialdemokratischen Führern nicht an. Im Grunde hoffen sie nur auf jene mittleren 
und unteren Schichten der Bourgeoisie - sowie auf die Monarchie bzw. auf das Offizierkorps -, die sie beschwören, anstelle des Faschismus wieder die „Demokratie“ „einzuführen “. Dafür stellen sie ihnen in Aussicht, ihrerseits den revolutionären Kräften des Proletariats die Spitze abzubrechen, sei es durch Beschwichtigung der Arbeiter durch eine Koalitionsregierung, sei es auch durch Unterdrückung jeder Regung im Proletariat mit Waffengewalt, wenn es notwendig sein sollte.....

[Internationale Pressekorrespondenz Nr. 122 vom 30. Oktober 1928, S. 2408f.]

23.11 .1928

28.

Mussolinis neue Gesetze Von Pietro (Wien).

Der italienische Senat hat in seiner Sitzung vom 16. November zwei Gesetzentwürfe sanktioniert: über die Machtbefugnisse des Großen Faschistenrates und über die „Magna Charta der Arbeit“.

Die faschistischen Blätter preisen die neuen Gesetze gewissermaßen als eine „Demokratisierung" der faschistischen Diktatur, durch die die faschistische Diktatur „ihren individuellen Charakter abstreift". Das ist natürlich eine Heuchelei. Das faschistische Terrorregime steckt längst nicht mehr in den Kinderschuhen des kleinbürgerlichen "Individualismus". Die neuen Gesetze sind nur die juristische „Einfügung in die Verfassung " einer längst de facto bestehenden Lage: der in Tausenden von Institutionen des bürgerlichen Staates verankerten grausamen Gewaltherrschaft der italienischen Großkapitalisten und Großgrundbesitzer und deren Vollzugsorgan, des Großen Faschistenrats, gegenüber der werktätigen Bevölkerung Italiens. Einer Gewaltherrschaft, die sogar im bürgerlichen Lager keine Opposition duldet. Das Gesetz über den Großen Faschistenrat stellt eine Erweiterung des bereits früher angenommenen Gesetzes über die Wahlreform dar. Wie bekannt, liegt der Kern dieser Reform in der Aufhebung der Kammer als wählbarer Körperschaft. Ihre Mitglieder werden eigentlich von den faschistischen Parteiorganen ernannt, da nach der Wahlreform dem Großen Faschistenrat das Recht zuerkannt wird, aus den von den faschistischen Organisationen vorgeschlagenen Namen für die Kammerwahlen die ihm genehmen Abgeordneten auszuwählen. Das neue Gesetz über die Machtbefugnisse des Großen Faschistenrates geht noch weiter. Es fügt den Großen Faschistenrat selbst in den korporativen Staat ein, als „Synthese aller Kräfte und aller Institutionen der Nation“. Das Gesetz bestimmt die Zusammensetzung des Großen Faschistenrates, dessen Mitglieder sich aus dem Regierungschef, aus dem Senats- und Kammerpräsidenten, den Ministern und Unterstaatssekretären und allen denjenigen rekrutieren, die "der Bedeutung ihrer Stellung als Führer des Faschismus und als wichtigste Exponenten der faschistischen Partei oder als führende Persönlichkeiten des Kulturlebens, der großen Organisationen und Institutionen darauf Anspruch haben können“. Dem großen Faschistenrat werden beschließende und beratende Rechte eingeräumt. Seine beschließenden Rechte betreffen die Vorbereitung der Kandidatenlisten für die Kammerwahlen. Seine beratenden Rechte beziehen sich in erster Reihe auf Verfassungsfragen, das Gesetz jedoch bemerkt dazu, „daß die Meinung des Großen Faschistenrates auch in diesen Fragen obligatorisch ist". Im Falle einer neuen Regierungs- 
bildung hat der Große Faschistenrat das Recht, dem König eine Liste derjenigen Persönlichkeiten zu unterbreiten, aus denen der neue Regierungschef auszuwählen ist. Die Machtbefugnisse des Großen Faschistenrates erstrecken sich auf alle Glieder des korporativen Staates: auf die Gewerkschaften, auf die Korporationen, auf die Kulturinstitutionen usw. Auch die Erziehung der Jugend fällt unter die Kompetenz des Rates. Er ist schließlich das höchste Organ der Faschistischen Partei selbst, das deren Politik bestimmt und alle Funktionäre der Partei ernennt.

Das neue Gesetz über die Machtbefugnisse des Großen Faschistenrates bildet zusammen mit der Wahlreform den würdigen Abschluß des Prozesses, der mit der "Korrigierung" der Ergebnisse der ersten Wahlen nach der faschistischen Machtergreifung (als die Opposition im Parlament eine starke Vertretung bekam) durch den Mord an Matteotti und die allmähliche Verdrängung aller nichtfaschistischen Abgeordneten aus dem Parlament begann und im November 1926 durch die Auflösung. und das Verbot aller nichtfaschistischen Parteien zusammen mit den freien Gewerkschaften fortgesetzt wurde. ....

[Internationale Pressekorrespondenz Nr. 131 rom 23. November 1928, S. 2593f.]

29.

Zum Internationalen Antifaschisten-Kongreß :

Febr. 1929

\section{Das Bündnis zwischen Faschismus und Vatikan}

..... Als das Initiativkomitee des bevorstehenden Internationalen Antifaschistenkongresses die Tagesordnung des Kongresses festsetzte, da war der Annäherungsprozeß zwischen dem Faschismus und dem Vatikan noch nicht zu einem Bündnis zwischen den beiden herangereift. In die Tagesordnung des Kongresses wurde die Untersuchung des Verhältnisses zwischen Rutenbündel und Krummstab auch nicht mitaufgenommen. Inzwischen ist aber Mussolini glücklich am Gegenpol seines ursprünglichen Standpunktes der Kirche gegenüber angelangt: auf seine Veranlassung hin wurde zwischen dem italienischen Staat und dem Vatikan ein Friedensvertrag von großer innenpolitischer und außenpolitischer Bedeutung unterschrieben [Lateranverträge vom 11. 2. 1929]. Diese Tatsache verpflichtet den Kongreß, auch dieser Frage besondere Beachtung zu schenken.

Die Einigung zwischen dem Faschismus und dem Vatikan ist auf folgender Grundlage zustande gekommen:

Im ersten Teile des Vertrages hebt der italienische Staat das sogenannte Garantiegesetz von 1871, in dem der Verzicht des Papstes auf den früheren Kirchenstaat und die Anerkennung des italienischen Königreiches mit Rom als Hauptstadt gefordert wurde, auf, und erkennt das Prinzip und die volle Macht und souveräne Hoheit des Papstes auf einem bestimmten Gebiet, genannt vatikanische Stadt, an. Italien zahlt eine Summe auch für die früheren kirchlichen Provinzen und für den Verlust der Kirchengüter; es schließt ein Konkordat über die Beziehungen zwischen der Kirche und dem italienischen Staat ab. Der päpstliche Stuhl erklärt zugleich endgültig die römische Frage für beigelegt und erkennt das italienische Königreich in seiner gegenwärtigen Gestalt und Verfassung an. Der zweite Teil der Vereinbarungen bezieht sich auf die Regelung der finanziellen Fragen. Als Rom von der bürgerlichen 
Revolution erobert wurde, bot der italienische Staat als Entschädigung dem Papst die jährliche Zahlung von $3 \frac{1}{2}$ Millionen Lire an. Die Vorgänger des jetzt regierenden Papstes haben aber diese Zuwendungen des „kirchenräuberischen “ Italiens abgelehnt. Jetzt soll dieser seit 1871 nicht erhobene Betrag mit Zinseszinsen ausgezahlt werden. Dies bedeutet eine ungeheure Belastung der italienischen Werktätigen: das Anziehen der Steuerschraube wird die unmittelbare Folge des faschistisch-päpstlichen Friedensschlusses sein. Der Vatikan seinerseits erhält damit einen großen neuen Kriegsfonds zur Führung der klerikalen Propaganda in der Welt.

Der dritte Teil der Vereinbarung enthält ein Konkordat zwischen dem päpstlichen Stuhl und dem italienischen Staat. Seine wichtigsten Bestimmungen beziehen sich auf die Auslieferung der Schulen an die Kirche, die Anerkennung aller konfessionellen Schulen als gleichberechtigt mit den staatlichen und auf die Neuregelung des Eherechtes, worunter wahrscheinlich die Abschaffung der Zivilehe und die obligatorische Einführung der kirchlichen Trauung zu verstehen ist. Mit einem Worte: Italiens gesamtes geistiges und kulturelles Leben wird verpfafft.

Noch wichtiger sind die imperialistischen Auswirkungen des Vertrages. Bisher war Frankreich die Macht, die das Protektorat über die katholischen Missionen ausübte: die katholischen Klöster waren die Agenturen der französischen Kolonialpolitik. Nun soll diese Rolle entweder vollkommen auf das „bekehrte“ Italien übergehen oder wenigstens die Funktion geteilt werden, wodurch die Kolonialpläne des italienischen Imperialismus einen mächtigen Impuls bekommen und die Kriegsgefahr verschärft wird.

Diese weltpolitische Auswirkung des Bündnisses zwischen dem Krummstab und dem Rutenbündel in Rom ist es, gegen die in dieser Frage die antiimperialistische Front in erster Reihe zu mobilisieren ist.

[Internationale Pressekorrespondenz Nr. 15 (Februar 1929), S. 293]

30.

Die Bedeutung des internationalen Antifaschistenkongresses

Von I. Silone (Lugano).

Die Bedeutung des Internationalen Antifaschisten-Kongresses, der am 9. und 10. März in Berlin stattfindet, ist in der Antwort enthalten, die die Kommunisten auf folgende Fragen geben:

An welchem Punkt ist der Faschismus angelangt? Welchen Faktor stellt er in der gegenwärtigen internationalen Lage dar? In welchem Maße und in welchen Ländern bestätigt das Erscheinen der faschistischen Gefahr unsere Perspektive der verschärften organischen Krise der kapitalistischen Gesellschaft?

Nur, nachdem wir diese Fragen beantwortet haben, ist es möglich, das Problem zu behandeln: Wie ist der Kampf gegen den Faschismus zu führen?

Der italienische reformistische Führer Turati definierte den Faschismus als „eine vorübergehende Erscheinung der Kriegspsychose“, und diese Definition wurde in verschiedenen Variationen von der Sozialdemokratie aller Länder wiederholt. Der Faschismus sollte also eine akute, heftige, aber kurzdauernde Krankheit sein, auf die eine Periode der Erneuerung folgt..... 
Die Entwicklung der Lage in Italien in den letzten sieben Jahren - vom Marsch auf Rom bis zum Konkordat zwischen dem Faschismus und dem Vatikan - ging langsam vor sich, aber stets im Gegensatz zu den Voraussagungen der Sozialdemokratie. Wenn wir den ganzen Entwicklungsgang ins Auge fassen, so können wir erklären, daß der Faschismus als die besondere Methode der Stabilisierung des Kapitalismus in Italien erscheint. Die Besonderheiten dieser Bestrebung zur Stabilisierung waren von der sozialen und wirtschaftlichen Struktur Italiens bestimmt: insbesondere vom großen spezifischen Gewicht der Arbeitskraft in der ganzen Organisation der italienischen Industrie und Landwirtchaft, sowie von dem Entwicklungsgrad der Arbeiterbewegung. Mit Hilfe des Faschismus gelang es dem italienischen Kapitalismus, sich die höchsten Profite in der Industrie und die höchste Bodenrente in der Landwirtschaft zu sichern, durch eine unerhörte Unterdrückung der Werktätigen. Mit Hilfe des Faschismus hat die italienische Bourgeoisie neue Organisationsformen der Industrie und des Bankwesens entwickelt, sie hat um die Hauptgruppen der Kapitalisten die kleinbürgerlichen Reserven gesammelt, sie hat einen Staatstypus geschaffen, wodurch der Staat sich jeder Kontrolle durch die werktätigen Massen entzieht und immer mehr mit den die Produktion beherrschenden Gruppen identisch wird. Der faschistische Staat greift selbst als entscheidender Faktor in alle wichtigen Momente der Produktion ein (Staatskapitalismus).

Auf politischem und sozialem Gebiet entspricht dieser Identifizierung des Faschismus mit der italienischen Bourgeoisie ein Prozeß der Verengerung der sozialen Basis des Faschismus. In den letzten sechs Monaten haben eine Reihe von politischen Ereignissen gezeigt, inwieweit die Loslösung der Mittelschichten vom Faschismus fortgeschritten ist und inwieweit der Faschismus sich auf den Block der Großbourgeoisie und der Agrarier stützt. . . . .

Die neuen Kräfteverhältnisse, die seit zwei Jahren zwischen den oppositionellen Parteien bestehen, die Tatsache, daß die Kommunistische Partei als Führerin des antifaschistischen Kampfes auch von vielen nicht proletarischen Elementen anerkannt wurde, die Tatsache, daß die Sozialdemokratie selbst dies anerkennen muß, führt zu der Folgerung: Der Kampf um den Sturz des Faschismus muß mit dem Kampf um den Sturz der kapitalistischen Gesellschaft zusammenfallen. Wer heute gegen den Faschismus kämpfen will, muß auf der Grundlage des Klassenkampfes gegen die Bourgeoisie kämpfen. Diese Erkenntnis hat manche „Freigeistige“ und manche „freien" Denker auf die Probe gestellt, die aus moralischen und ideologischen Gründen Antifaschisten sind. Sie verheimlichen es nicht, daß sie vor der Tatsache, daß nach dem Faschismus einzig und allein das revolutionäre Proletariat an die Macht kommen kann, sie in Zweifel darüber geraten, ob sie den Faschismus bekämpfen sollen.

Es gibt sicherlich auch im Ausland solche "freigeistigen" Leute und „sentimentale“ Menschen, denen es zwar vor den Schrecken des Faschismus graut, die aber die Bedeutung des realen politischen Kampfes, der in Italien vor sich geht, nicht erkennen. Erkennen müssen ihn aber die Arbeiter der übrigen Länder.

Die falsche Auffassung des italienischen Faschismus durch die Sozialdemokratie verdeckt vor den Augen der Arbeiter die Keime des Faschismus, die in vielen anderen, noch „demokratisch" regierten Ländern aufgehen. Der Internationale AntifaschistenKongreß darf sich nicht darauf beschränken, gegen den sich an der Macht befindenden Faschismus zu kämpfen, sondern er muß die Arbeiter warnen und ihnen die Elemente 
des Faschismus zeigen, die in der bürgerlichen Politik enthalten sind und in einer gewissen Situation (wie in Österreich) in allen Ländern gefährlich werden können.

Eine klare Erkenntnis der faschistischen Gefahr wird für viele revolutionäre Arbeiter durch die falsche Charakterisierung des Faschismus erschwert. Man fällt von einem Extrem ins andere: die einen sehen jede Gewaltaktion der Bourgeoisie und des bürgerlichen Staates für Faschismus an, die anderen glauben, daß der Faschismus eine Besonderheit der rückständigen kapitalistischen Länder sei. Wenn wir den Massen die faschistische Gefahr klarmachen wollen, so müssen wir auf die Keime des Faschismus in den einzelnen Ländern hinweisen.

[Internationale Pressekorrespondenz Nr. 23 (März 1929), S. 487] 